\title{
HOMOGENEOUS RIEMANIIAN MANIFOLDS OF NEGATIVE CURVATURE
}

\author{
BY SHOSHICHI KOBAYASHI \\ Communicated by R. Bott, March 21, 1962
}

In [1], Wolf proved that a homogeneous Riemannian manifold of constant negative curvature is necessarily simply connected. The purpose of this paper is to prove the following generalization.

TheOREM. Let $M$ be a homogeneous Riemannian manifold possessing the following properties:

(1) The sectional curvature is nonpositive everywhere on $M$;

(2) For every nonzero tangent vector of $M$, there exists a tangent plane with negative sectional curvature which contains it.

Then $M$ is simply connected.

As an immediate consequence, we have

Corollary. Let $M^{*}$ be a simply connected Riemannian symmelric space whose components in the De Rham decomposition are all noncompact and non-Euclidean. If $D$ is a properly discontinuous group of isometries of $M^{*}$ such that $M=M^{*} / D$ is homogeneous, then $D$ contains only the identity element.

Proof of Theorem. Let $M^{*}$ be the universal covering space of $M$ with the naturally induced Riemannian structure, so that $M=M^{*} / D$ where $D$ is a properly discontinuous group of isometries acting freely on $M^{*}$. Let $p: M^{*} \rightarrow M$ be the covering projection. Let $x$ be an arbitrary point of $M$. Assuming that $D$ is nontrivial, let $x_{0}^{*}$ and $x_{1}^{*}$ be two distinct points of $M^{*}$ such that $x=p\left(x_{0}^{*}\right)=p\left(x_{1}^{*}\right)$. Let $\tau^{*}=x_{t}^{*}$, $0 \leqq t \leqq 1$, be a geodesic from $x_{0}^{*}$ to $x_{1}^{*}$ where $t$ is an affine parameter; $\tau^{*}$ is unique if $M$ possesses the property (1). Let $\tau=x_{t}, 0 \leqq t \leqq 1$, be the geodesic in $M$ defined by $x_{t}=p\left(x_{t}^{*}\right)$ so that $x=x_{0}=x_{1}$.

Lemma. If $M$ is a homogeneous Riemannian manifold with the property (1), then $\tau$ is an orbit of a 1-parameter group of isometries so that the closed geodesic $\tau$ is smooth even at $x=x_{0}=x_{1}$.

Proof of Lemma. Let $X$ be an infinitesimal isometry of $M$ tangent to $\tau$ at $x_{0}$. Then $X$ is a Jacobi field along $\tau$. At each point of $\tau$ we decompose $X$ into a tangent vector and a normal vector to $\tau$ so that we have $X=Y+Z$ where $Y$ (resp. $Z$ ) is a Jacobi field along $\tau$ tangent to $\tau$ (resp. normal to $\tau$ ). Since $Z$ vanishes at $x_{0}=x_{1}$ and since the curvature of $M$ is nonpositive, $Z$ vanishes at every point of $\tau$. This 
proves that $X$ is tangent to $\tau$ at each point, thus completing the proof of Lemma.

To complete the proof of Theorem, let $V$ be any infinitesimal isometry of $M$. We define a non-negative function $f(t),-\infty \angle t \angle \infty$, as follows:

$f(t)=$ the square of the length of $V$ at the point $x_{t}$ for $0 \leqq t \leqq 1$, and then we extend it to a periodic function of period 1. By Lemma, $f(t)$ is differentiable for all values of $t$.

Let $X$ be the vector field along $\tau$ tangent to $\tau$; the infinitesimal isometry in the proof of Lemma serves our purpose. Let $V^{\prime}$ and $V^{\prime \prime}$ be the first and the second covariant derivatives of $V$ in the direction of $X$. Let $g$ and $R$ be the metric and the curvature tensor fields of $M$ respectively. Then we have, for $0 \leqq t \leqq 1$,

$$
\begin{aligned}
f(t) & =g(V, V)_{x_{t}}, \quad f^{\prime}(t)=2 \cdot g\left(V^{\prime}, V\right)_{x_{t}}, \\
f^{\prime \prime}(t) & =2 \cdot g\left(V^{\prime}, V^{\prime}\right)_{x_{t}}+2 \cdot g\left(V^{\prime \prime}, V\right)_{x_{t}} \\
& =2 \cdot g\left(V^{\prime}, V^{\prime}\right)_{x_{t}}-2 \cdot g(R(V, X) X, V)_{x t} \geqq 0 .
\end{aligned}
$$

Since $f(t)$ is a periodic differentiable function and since $f^{\prime \prime}(t) \geqq 0, f(t)$ is a constant function. Hence, $f^{\prime \prime}(t)=0$. In particular, $g\left(V^{\prime}, V^{\prime}\right)=0$ and $g(R(V, X) X, V)=0$.

On the other hand, if $M$ is a homogeneous Riemannian manifold with the property (2), then there exists an infinitesimal isometry $V$ of $M$ such that $g(R(V, X) X, V)_{x_{0}}<0$. This contradiction comes from the assumption that $D$ is nontrivial. Q.E.D.

We conclude the paper by posing three questions. (a) Can we replace (2) in Theorem by the assumption that $M^{*}$ has no Euclidean part in the De Rham decomposition? (b) Is every homogeneous Kaehler manifold with negative holomorphic sectional curvature simply connected? (c) Is every homogeneous Riemannian manifold with negative Ricci tensor simply connected?

Clearly, the affirmative answer to (c) will imply our theorem and the affirmative answer to (b). Every homogeneous bounded domain in $\mathbf{C}^{n}$ is an Einstein-Kaehler manifold with negative Ricci tensor; we do not know even in this case whether the manifold is simply connected or not.

\section{BIBLIOGRAPHY}

1. J. A.Wolf, Sur la classification des variêtés riemanniennes homogènes à courbure constante, C. R. Acad. Sci. Paris 250 (1960), 3443-3445.

University of British Columbia 